\title{
A STATISTICAL TREATMENT OF THE STELLAR ROTATIONAL VELOCITIES WHICH CONSIDERS THE BREAK-UP LIMIT
}

\author{
P. L. BERNACCA \\ Asiago Astrophysical Observatory, University of Padua, Italy
}

\begin{abstract}
This paper develops a new statistical approach to the study of the axial rotational velocities of the stars, by considering the break-up limit or any upper limit for the equatorial rotational velocities. The main conclusions are as follows: (1) For random orientation of the axis of rotation, the frequency function of the inclination $\alpha$ to the line of $\operatorname{sight}$ is not $\sin \alpha$, as assumed until now. (2) For stars with the same break-up velocity $v_{b}$, the distribution of $\alpha$ is given by:

$$
\psi(\alpha)=v_{b} \sin \alpha \int_{0}^{v_{b} \sin \alpha} f(y)\left(v_{b}^{2}-y^{2}\right)^{-1 / 2} \mathrm{~d} y,
$$

where $f(y)$ is the distribution of the apparent velocities $y=v \sin \alpha, v$ being the true rotational velocity. New integral equations governing the frequency function of $\alpha, y$ and $v$ have been derived and the correct procedure to treat observations has been discussed; a simple method is also suggested to get an approximate trend of the distribution of the true velocities directly from the observed histogram. The method of analysis proposed in this paper has been applied to a re-discussion of the rotational behaviour of a group of Be stars.
\end{abstract}

\section{Introduction}

It is well known that the apparent (equatorial) rotational velocity of a star can be determined spectroscopically (Shajn and Struve, 1929; Slettebak, 1949; Huang, 1953). If the axis of rotation of a star is inclined at an angle $\alpha$ to the line of sight, the apparent velocity $y$ is given by:

$$
y=v \sin \alpha,
$$

where $v$ is the true (equatorial) rotational velocity.

Since the value of $\alpha$ for individual stars is unknown, one can attempt only to derive group properties, such as the frequency distribution of $v$, from observations of $y$ in a sample of stars and this statistical approach requires some assumption as to the frequency of occurrence of a given angle of inclination among the stars in the sample under consideration. The hypothesis usually made is that the axes of rotation are randomly distributed in space so that the function

$$
\psi(\alpha)=\sin \alpha
$$

may be considered to represent the distribution of $\alpha$. Let us recall here briefly the main points of the theory as developed, for instance, by Chandrasekhar and Münch (1950).

The integral equations:

$$
f(y)=y \int_{y}^{\infty} \frac{\varphi(v) \mathrm{d} v}{v\left(v^{2}-y^{2}\right)^{1 / 2}}
$$




$$
\varphi(v)=-\frac{2}{\pi} v^{2} \frac{\partial}{\partial v} v \int_{v}^{\infty} \frac{f(y) \mathrm{d} y}{y^{2}\left(y^{2}-v^{2}\right)^{1 / 2}}
$$

relate the frequency function $\varphi(v)$ of the true velocities with the corresponding $f(y)$ of the apparent ones. Since the differentiation of an observed distribution (given often in the form of a histogram) can lead to results which are misleading, instead of deriving $\varphi(v)$ by $(4)$, the function:

$$
\varphi(v)=\frac{j}{\sqrt{ } \pi}\left[e^{-j^{2}\left(v-v_{1}\right)^{2}}+e^{-j^{2}\left(v+v_{1}\right)^{2}}\right]
$$

is assumed and the problem is solved by appropriate selection of the parameters $j$ and $v_{1}$ to fit the computed distribution $f(y)$ to the observed histogram.

The central moments of $\varphi(v)$ can be derived by means of (3) without the knowledge of the function itself; for instance, the mean and the mean square deviation are given respectively by:

$$
\begin{aligned}
\langle v\rangle & =\frac{4}{\pi}\langle y\rangle \\
\left\langle(v-\langle v\rangle)^{2}\right\rangle & =1.5\left\langle y^{2}\right\rangle-\frac{16}{\pi^{2}}\langle y\rangle^{2}
\end{aligned}
$$

so that we can compute them directly from the observations.

In the above analysis no restrictions are imposed on the variables $y$ and $v$ (except of course $y \leqslant v$ ) and relation (2) is an 'a priori' assumption, which is considered to be valid for any observed random sample of rotating stars. Since the condition of stability requires that a star rotates with an equatorial velocity not greater than its break-up velocity $v_{b}$ (determined for each star by radius and mass distribution), it follows that any statistical analysis must exclude the expectation of stars rotating with a velocity greater than some known limit; in particular, if a sample of stars with the same break-up velocity is considered, any $\varphi(v)$ must vanish for $v>v_{b}$ and $\langle v\rangle$ must not exceed $v_{b}$ when formula (6) is used.

The question arises now whether the foregoing procedure will yield always correct results from this point of view; let us consider a sample of stars whose rotational velocities have a unique upper limit (in particular the same break-up velocity) and suppose that the observed frequency distribution be $f_{0}(y)$ :

If one assumes a function $\varphi\left(v ; v_{1}, j\right)$ so that:

$$
\begin{aligned}
\varphi\left(v ; v_{1}, j\right) & =\varphi_{0}\left(v ; v_{1}, j\right) & & 0 \leqslant v \leqslant v_{b} \\
& =0 & & v_{b}<v
\end{aligned}
$$

it may be possible to fit $f\left(y ; v_{1}, j\right)$, as computed by (3), to $f_{0}(y)$; however this is not true in general. Let us consider the following two cases:

(i) When a distribution:

$$
f_{1}(y)=\frac{y}{v_{b}\left(v_{b}^{2}-y^{2}\right)^{1 / 2}} \quad 0 \leqslant y \leqslant v_{b}
$$


is observed, a Dirac $\delta$-function $\delta\left(v-v_{b}\right)$ is the solution of the integral Equation (3), meaning that all stars rotate at the equatorial break-up velocity. Formula (6) gives, of course, $\langle v\rangle=v_{b}$ when $\langle y\rangle$ has been computed by (8). On the other hand, for a frequency function $f_{2}(y)$, so that $f_{2}(y)<f_{1}(y)$ in a given interval $\left(0, y_{0}\right)$, it is impossible to find a true distribution $\varphi(v)$ vanishing for $v>v_{b}$ : it is obvious that (6) yields in this case $\langle v\rangle\rangle v_{b}$.

It can be argued that if $f_{2}(y)$ is observed, the hypothesis of random orientation for the rotational axes is no longer valid but at least two conclusions can be drawn: first, one must care about the assumption 'a priori' of (2); second, the inclusion of $v_{b}$ gives the possibility of detecting deviation from randomness.

(ii) Let us suppose that all apparent velocities in a sample have been observed to lie between $y_{0}$ and $v_{b}$. Since $v<v_{b}$, relation (1) states that $\sin ^{-1}\left(y_{0} / v_{b}\right) \leqslant \alpha \leqslant \pi / 2$ for each star. If the axes of rotation are randomly distributed, with $\alpha$ within the above limits, it may be assumed now 'a priori' (see Figure 2):

$$
\begin{array}{rlr}
\psi(\alpha) & =\begin{array}{cr}
v_{b} \sin \alpha & \\
\left(v_{b}^{2}-y_{0}^{2}\right)^{1 / 2} &
\end{array} \\
& =0 & 0 \leqslant \alpha \sin ^{-1}\left(y_{0} / v_{b}\right) \\
& 0 \leqslant \sin ^{-1}\left(y_{0} / v_{b}\right) .
\end{array}
$$

The integral Equation (3) and (4) need to be modified as well as formulae (6) and (7). It follows that (2) is not always the correct frequency function for the inclination $\alpha$. Further, an example analogous to case (i) can be easily constructed by means of (9) and the modified integral equations, which would show how the assumption 'a priori' of (9) may be not quite correct.

In the following sections we attempt to derive a general frequency distribution $\psi(\alpha)$ and develop thereby a different method of analysis; therefore, a conclusive judgment on the statistical meaning of (2) will be given later.

\section{The Distribution Function of the Inclination of the Rotational Axes}

Let us consider a sample of stars of known break-up velocity $v_{b}$ and whose apparent velocities $y$ have been determined: by (1) and the condition $v \leqslant v_{b}$, we have for each star:

$$
y \leqslant v_{b} \sin \alpha .
$$

If an angle $\theta$ is defined by the relation

$$
y=v_{b} \sin \theta
$$

the inclination $\alpha$ of each star must satisfy the condition:

$$
\theta \leqslant \alpha \leqslant \pi / 2 \text {. }
$$

Since $y$ may range from 0 to one of the values $v_{b}, \theta$ will vary between 0 and $\pi / 2$. In the $(\theta, \alpha)$-plane the representative points of the stars lie in the triangle defined by the straight lines $\theta=0, \alpha=\pi / 2$ and $\alpha=\theta$ (Figure 1). The number of stars $\mathrm{d} n$ with $\theta$ and $\alpha$ 
within $(\theta, \theta+\mathrm{d} \theta)$ and $(\alpha, \alpha+\mathrm{d} \alpha)$ can be written:

$$
\mathrm{d} n=N \psi(\theta, \alpha) \mathrm{d} \theta \mathrm{d} \alpha,
$$

where $N$ is the total number of stars and $\psi(\theta, \alpha)$ is the distribution function in the ( $\theta$, $\alpha)$-plane.

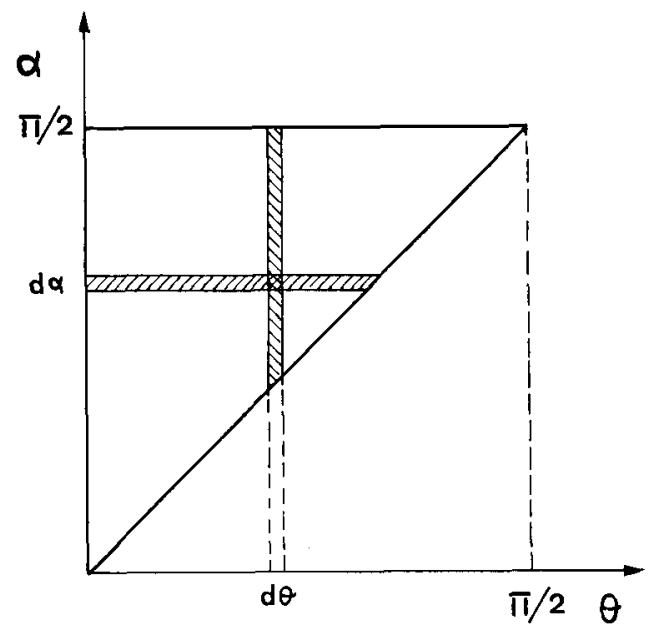

Fig. 1. The triangle defines the region occupied by the representative points $(\theta, \alpha)$ of stars rotating with an inclination $\alpha$ to the line of sight and whose apparent velocity is $y=v_{b} \sin \theta, v_{b}$ being the break-up limit.

The bivariate distribution $\psi(\theta, \alpha)$ can be written (see e.g. Trumpler and Weaver, 1953) as:

$$
\psi(\theta, \alpha)=\psi_{\theta}(\theta) \psi(\alpha \mid \theta)
$$

where $\psi_{\theta}(\theta)$ is the marginal distribution of the sample with respect to $\theta$, which is known from the observations, while $\psi(\alpha \backslash \theta)$ is the frequency function of $\alpha$ for each $\theta$-array. If one picks from the sample all stars with a given $\theta$ and assumes that the axes of rotation of these stars are randomly distributed in space with the condition (12), it follows (see Figure 2):

$$
\psi(\alpha \mid \theta) \mathrm{d} \alpha=\frac{2 \pi \sin \alpha \mathrm{d} \alpha}{2 \pi-2 \pi(1-\cos \theta)}=\frac{\sin \alpha}{\cos \theta} \mathrm{d} \alpha \quad \alpha \geqslant \theta .
$$

Thus the bivariate distribution (14) is known and the marginal distribution of the sample with respect to $\alpha, \psi(\alpha)$, can be obtained simply by:

$$
\psi(\alpha)=\int_{0}^{\alpha} \psi(\theta, \alpha) \mathrm{d} \theta=\sin \alpha \int_{0}^{\alpha} \frac{\psi_{\theta}(\theta)}{\cos \theta} \mathrm{d} \theta .
$$

If only stars with the same break-up velocity are considered (or if only one upper limit to the true velocities for all stars does exist), the frequency function of $\theta$ can be written 


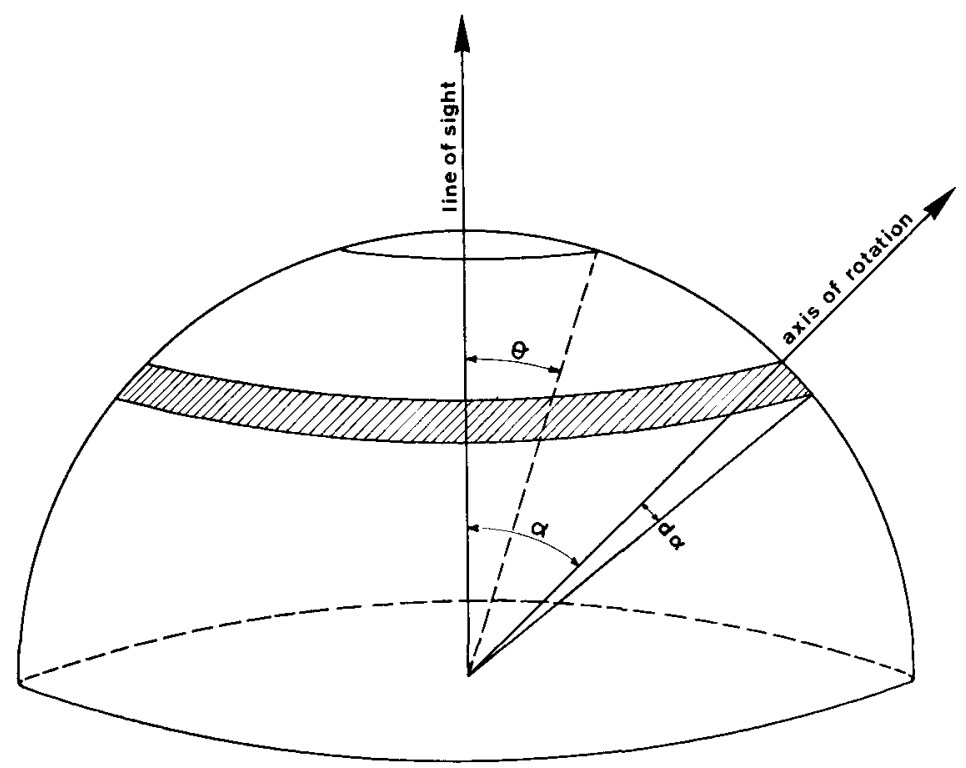

Fig. 2. The probability of occurrence of $\alpha$ (ranging from $\theta$ to $\pi / 2$ ), between $\alpha$ and $\alpha+\mathrm{d} \alpha$, is given by $\sin \alpha \mathrm{d} \alpha / \cos \theta$.

as:

$$
\psi_{\theta}(\theta)=v_{b} f\left(v_{b} \sin \theta\right) \cos \theta
$$

so that, by means of (16), we have:

$$
\psi(\alpha)=v_{b} \sin \alpha \int_{0}^{\alpha} f\left(v_{b} \sin \theta\right) \mathrm{d} \theta
$$

or

$$
\psi(\alpha)=v_{b} \sin \alpha \int_{0}^{v_{b} \sin \alpha} \frac{f(y) d y}{\left(v_{b}^{2}-y^{2}\right)^{1 / 2}} .
$$

Relations (16) and (18) or (19) represent the distribution of the inclination of the rotational axes; they depend on the observations through $\psi_{\theta}(\theta)$ or $f(y)$.

The statistical meaning of (2) and (9) now becomes clear, since they can be derived from (19) if particular observations such as $f(y)=\delta(y)$ and $f(y)=\delta\left(y-y_{0}\right)$ are considered respectively: the early function $\psi(\alpha)=\sin \alpha$ [and (9) too] appears to be only an array distribution and it can no longer be used in the rotational context, unless some empirical information exists showing that the fraction of stars with $\alpha$ between $\alpha$ and $\alpha+\mathrm{d} \alpha$ is just $\sin \alpha \mathrm{d} \alpha$.

\section{The Moments of the Distribution of the True Rotational Velocities}

The mean, the mean square deviation and the skewness of $\varphi(v)$ can be derived from 
the general relation:

$$
\left\langle y^{n}\right\rangle=\left\langle v^{n}\right\rangle \int_{0}^{\pi / 2}(\sin \alpha)^{n} \psi(\alpha) \mathrm{d} \alpha .
$$

Let $A_{n}$ be the integral factor contained in (20); it then follows that:

$$
\begin{aligned}
\langle v\rangle & =A_{1}^{-1}\langle y\rangle \\
\left\langle(v-\langle v\rangle)^{2}\right\rangle & =A_{2}^{-1}\left\langle y^{2}\right\rangle-A_{1}^{-2}\langle y\rangle^{2} \\
\left\langle(v-\langle v\rangle)^{3}\right\rangle & =A_{3}^{-1}\left\langle y^{3}\right\rangle-3\left(A_{1} A_{2}\right)^{-1}\langle y\rangle\left\langle y^{2}\right\rangle+2 A_{1}^{-3}\langle y\rangle^{3} .
\end{aligned}
$$

Hereafter we consider only the case of stars with the same break-up velocity, so that (21) may be used to compute the mean true velocity for stars of known apparent velocity, having approximately the same spectral type and luminosity class; however the following results may be applied to any sample of rotating stars whenever a unique upper limit has been recognized. Thus the $A_{n}$ are obtained from (20) using the distribution (18) or (19).

Any observed distribution, given in the form of a histogram, can be written as:

$$
f(y)=I_{r}=\frac{N_{r}}{N\left(y_{r}-y_{r-1}\right)} \quad y_{r-1} \leqslant y \leqslant y_{r}
$$

with $r=1,2, \ldots k$, where $k$ is the number of intervals in which $\left(0, v_{b}\right)$ is divided, $N$ is the total number of stars, $N_{r}$ is the number of stars whose apparent velocity satisfies the condition $y_{r-1}<y<y_{r}$; further, in formula (24), $y_{0}=0$ and $y_{k}=v_{b}$.

Since the $N_{r}$ stars may be considered uniformly distributed in $\left(y_{r-1}, y_{r}\right)$, their distribution function $f_{r}(y)$ is given by:

$$
\begin{aligned}
f_{r}(y) & =1 /\left(y_{r}-y_{r-1}\right) & & y_{r-1} \leqslant y \leqslant y_{r} \\
& =0 & & \text { elsewhere }
\end{aligned}
$$

or, by means of (24):

$$
\begin{aligned}
f_{r}(y) & =I_{r} N / N_{r} & & y_{r-1} \leqslant y \leqslant y_{r} \\
& =0 & & \text { elsewhere }
\end{aligned}
$$

Each $f_{r}(y)$ produces a distribution function $\psi_{r}(\alpha)$, that, through (18) or (19), becomes:

$$
\psi_{r}(\alpha)=\left[\begin{array}{ll}
0 & 0 \leqslant \alpha \leqslant \theta_{r-1} \\
v_{b}\left(\alpha-\theta_{r-1}\right) \frac{I_{r} N}{N_{r}} \sin \alpha & \theta_{r-1} \leqslant \alpha \leqslant \theta_{r}, \\
v_{b}\left(\theta_{r}-\theta_{r-1}\right) \frac{I_{r} N}{N_{r}} \sin \alpha & \theta_{r} \leqslant \alpha \leqslant \pi / 2
\end{array}\right.
$$

where $\theta_{r}$ is defined by $y_{r}=v_{b} \sin \theta_{r}$.

From the definition of a distribution function, it can be shown easily that the total distribution $\psi(\alpha)$ for the sample of the $N$ stars, is given by:

$$
\psi(\alpha)=N^{-1} \sum_{1}^{k} N_{r} \psi_{r}(\alpha)
$$


so that, by (20), (27) and (28), we have for the quantities $A_{n}$ :

$$
A_{n}=v_{b} \sum_{1}^{k} I_{r}\left[\int_{\theta_{r-1}}^{\theta_{r}}\left(\alpha-\theta_{r-1}\right)(\sin \alpha)^{n+1} \mathrm{~d} \alpha+\left(\theta_{r}-\theta_{r-1}\right) \int_{\theta_{r}}^{\pi / 2}(\sin \alpha)^{n+1} \mathrm{~d} \alpha\right] \text { (29) }
$$

Taking $n=1,2,3$ successively, after integration and some reductions, (29) gives:

$$
\begin{aligned}
& A_{1}=\frac{v_{b}}{4} \sum_{1}^{k} I_{r}\left[\sin ^{2} \theta_{r}-\sin ^{2} \theta_{r-1}+\pi\left(\theta_{r}-\theta_{r-1}\right)-\left(\theta_{r}^{2}-\theta_{r-1}^{2}\right)\right] \\
& A_{2}=\frac{2}{3}+\frac{v_{b}}{9} \sum_{1}^{k} I_{r}\left(\sin ^{3} \theta_{r}-\sin ^{3} \theta_{r-1}\right) \\
& A_{3}=\frac{3}{4} A_{1}+v_{b} \sum_{16}^{k} I_{r}\left(\sin ^{4} \theta_{r}-\sin ^{4} \theta_{r-1}\right),
\end{aligned}
$$

where for $A_{2}$ the definition of $I_{r}$ and $\theta_{r}$ has been taken into account.

The quantities $A_{n}(n=1,2,3)$ appear to depend on the value of the histogram $I_{r}$ in the interval $r$ and on the particular subdivision of $\left(0, v_{b}\right)$, which defines each $\theta_{r}$. Although some general rules must be followed in the drawing of a histogram, it will depend somewhat on the choice of the observer. In order to illustrate the procedure and to test the personal choice of a histogram, we consider the rotational velocities

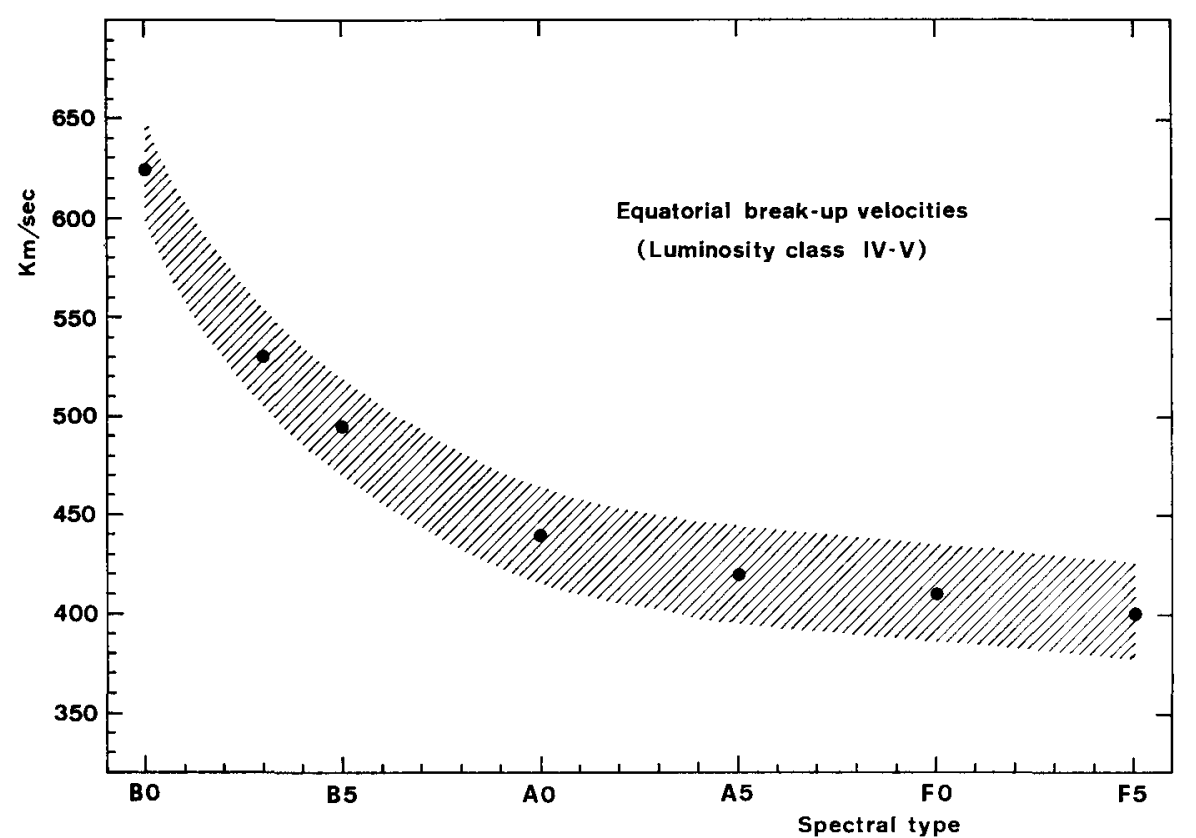

Fig. 3. Slettebak's computed break-up velocities against spectral type (full points). The uncertainty is of the order of $25 \mathrm{~km} / \mathrm{sec}$ (shaded area). 
determined by Slettebak (1966a) for 42 B6-B9e stars of luminosity class IV-V.

In Figure 3 the relationship between spectral type and break-up velocity is shown according to Slettebak (1966b); the shaded region represents the uncertainty in $v_{b}$ which is of the order of $25 \mathrm{~km} / \mathrm{sec}$. The break-up limit for B6-B9 stars ranges roughly from $480 \mathrm{~km} / \mathrm{sec}$ to $450 \mathrm{~km} / \mathrm{sec}$, but it may be lowered for Be stars if they are considered to be evolved objects. In the following a limit of $450 \mathrm{~km} / \mathrm{sec}$ is assumed for all the 42 stars, an approximation that is sufficient for our aim.

In Table I three possible frequency functions are given, computed by means of (24), together with $\theta_{r}$ and $\sin \theta_{r}$; the subdivision of $\left(0, v_{b}\right)$ in $k$ intervals has been made according to the first column of Table I and histogram (b) has been constructed with the condition $y_{r-1} \leqslant y<y_{r}$ for all but one star, whose velocity of $400 \mathrm{~km} / \mathrm{sec}$ has been arbitrarily lowered. The value of $A_{n}(n=1,2,3)$ is practically the same for histogram (a) and (c) while it is a little higher for case (b). According to formulae (21)-(23) we need first $\langle y\rangle$ and the higher moments of $f(y)$ : if they are derived by means of the histogram itself, the results are slightly different in the three cases (Table II, column (i)); if the observed moments are computed directly from the values of the apparent rotational velocities, no differences occur (Table II, column (ii)).

These results encourage one to believe that different histograms will yield with

TABLE I

Three possible empirical distributions $\left(I_{r}\right)$ for the same sample of Be stars (Slettebak, 1966a)

\begin{tabular}{|c|c|c|c|c|c|c|}
\hline$r$ & $\left(y_{r-1}, y_{r}\right)$ & $y_{r}-y_{r-1}$ & $N_{r}$ & $10^{2} I_{r}$ & $\theta_{r}$ & $\sin \theta_{r}$ \\
\hline \multicolumn{7}{|c|}{ (a) } \\
\hline 1 & $(0,25)$ & 25 & 0 & 0 & 0.05558 & 0.05556 \\
\hline 2 & $(25,75)$ & 50 & 2 & 0.0952 & 0.16745 & 0.16667 \\
\hline 3 & $(75,125)$ & 50 & 1 & 0.0476 & 0.28148 & 0.27778 \\
\hline 4 & $(125,175)$ & 50 & 3 & 0.1428 & 0.39942 & 0.38889 \\
\hline 5 & $(175,225)$ & 50 & 3 & 0.1428 & 0.52360 & 0.50000 \\
\hline 6 & $(225,275)$ & 50 & 10 & 0.4761 & 0.65746 & 0.61111 \\
\hline 7 & $(275,325)$ & 50 & 9 & 0.4285 & 0.80700 & 0.72222 \\
\hline 8 & $(325,375)$ & 50 & 13 & 0.6190 & 0.98511 & 0.83333 \\
\hline 9 & $(375,425)$ & 50 & 1 & 0.0476 & 1.23590 & 0.94444 \\
\hline \multicolumn{7}{|c|}{ (b) } \\
\hline 1 & $(0,100)$ & 100 & 2 & 0.0476 & 0.22410 & 0.22222 \\
\hline 2 & $(100,200)$ & 100 & 5 & 0.1190 & 0.46055 & 0.44444 \\
\hline 3 & $(200,300)$ & 100 & 13 & 0.3095 & 0.72973 & 0.66667 \\
\hline 4 & $(300,400)$ & 100 & 22 & 0.5238 & 1.09491 & 0.88889 \\
\hline \multicolumn{7}{|c|}{ (c) } \\
\hline 1 & $(0,50)$ & 50 & 0 & 0 & 0.11134 & 0.11111 \\
\hline 2 & $(50,130)$ & 80 & 3 & 0.0892 & 0.29310 & 0.28889 \\
\hline 3 & $(130,220)$ & 90 & 6 & 0.1587 & 0.51081 & 0.48889 \\
\hline 4 & $(220,280)$ & 60 & 10 & 0.3968 & 0.67158 & 0.62222 \\
\hline 5 & $(280,330)$ & 50 & 9 & 0.4285 & 0.82321 & 0.73333 \\
\hline 6 & $(330,360)$ & 30 & 13 & 1.0317 & 0.92730 & 0.80000 \\
\hline 7 & $(360,430)$ & 70 & 1 & 0.0340 & 1.27153 & 0.95556 \\
\hline 8 & $(430,450)$ & 20 & 0 & 0 & 1.57079 & 1.00000 \\
\hline
\end{tabular}




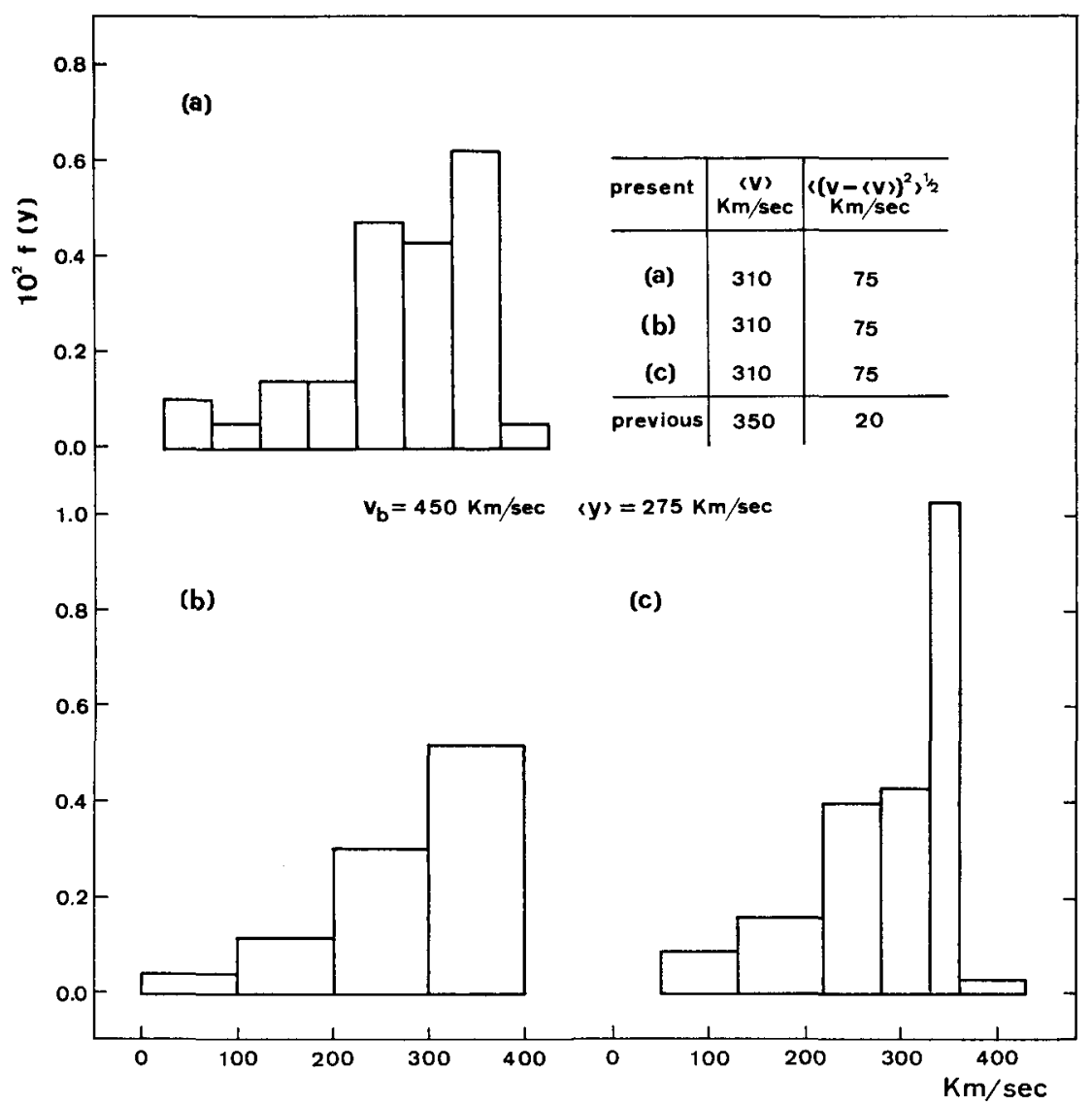

Fig. 4. (a), (b), (c). Three possible empirical distributions for the same group of stars. Inset: comparison between the central moments of the distribution of the true rotational velocities computed by the present method and the previous one. The case is the one of the Be stars studied by Slettebak (1966a).

sufficient accuracy the same information for a given sample of stars. One must bear in mind that the variety of histograms for the same sample is not large, since the size of the intervals $\left(y_{r-1}, y_{r}\right)$ can be neither too small nor too large, in order to avoid accidental errors or loss of information, respectively. In Figure 4 the three histograms of Table I have been drawn together with the present values of the first two central moments of $\varphi(v)$ and the corresponding ones determined by (6) and (7) (previous): the mean true velocity is $310 \mathrm{~km} / \mathrm{sec}$ instead of $350 \mathrm{~km} / \mathrm{sec}$ and the resulting root mean square deviation is much larger than the previous one: $75 \mathrm{~km} / \mathrm{sec}$ instead of $20 \mathrm{~km} / \mathrm{sec}$.

\section{The Distribution Function of the True Rotational Velocities for Homogeneous Samples}

Hereafter we discuss the method of deriving a true distribution only for homogeneous 
TABLE II

The mean and the root mean square of the true velocities $v$ derived for the distribution of Table $\mathbf{I}$ : (i) by $\langle y\rangle$ and $\left\langle y^{2}\right\rangle$ given by the histogram and (ii) by $\langle y\rangle$ and $\left\langle y^{2}\right\rangle$ computed directly from the observations

\begin{tabular}{llllllllll}
\hline Type & $A_{1}$ & $A_{2}$ & \multicolumn{1}{c}{$A_{3}$} & $\begin{array}{l}\text { (i) } \\
\langle y\rangle\end{array}$ & $\langle v\rangle$ & $\sigma_{v}$ & $\begin{array}{l}\text { (ii) } \\
\langle y\rangle\end{array}$ & $\langle v\rangle$ & $\sigma_{v}$ \\
\hline a & 0.88489 & 0.79983 & 0.73308 & 270 & 305 & 85 & 275 & 310 & 75 \\
b & 0.89112 & 0.81010 & 0.74626 & 280 & 315 & 90 & 275 & 310 & 75 \\
c & 0.88499 & 0.79935 & 0.73229 & 270 & 310 & 80 & 275 & 310 & 75 \\
\hline
\end{tabular}

$\sigma_{v}=\left\langle(v-\langle v\rangle)^{2}\right\rangle^{1 / 2}$

samples, i.e. for samples of stars which have the same equatorial break-up velocity. However, the method can be used for stars in general whenever an upper limit has been recognized, aside from the concept of break-up.

\section{A. FORMAL SOLUTION}

The true rotational velocity $v$, can be expressed, according to (1) and (11) as:

$$
v=\frac{v_{b} \sin \theta}{\sin \alpha} \quad \alpha \geqslant \theta \text {, }
$$

where $v_{b}$ is now a constant. The function $\varphi(v)$ can be obtained from the bivariate frequency function $\psi(\theta, \alpha)$ (Section 2 ) as follows:

$$
\varphi(v)=\int_{0}^{\sin ^{-1}\left(v / v_{b}\right)} \psi[\alpha(v, \theta), \theta]\left|\frac{\partial \alpha(v, \theta)}{\partial v}\right| \mathrm{d} \theta .
$$

Using relations (14), (15), (18) and (33) in (34), results in:

$$
\varphi(v)=\frac{v_{b}}{v^{2}} \int_{0}^{v} \frac{y^{2} f(y) \mathrm{d} y}{\sqrt{\left(v^{2}-y^{2}\right) \sqrt{\left(v_{b}^{2}-y^{2}\right)}}} .
$$

The integral Equation (35) can be easily solved with respect to $f(y)^{*}$ in order to

* Relation (35) is a Volterra's equation of the first kind which may be written

$$
v \varphi(v)=\int_{0}^{v} \frac{v_{b} y^{2} f(y)}{\sqrt{\left(v_{b}^{2}-y^{2}\right)}} \frac{\mathrm{d} y}{v \sqrt{\left(v^{2}-y^{2}\right)}} .
$$

Since the kernel $k(y, v)=1 / v \sqrt{ }\left(v^{2}-y^{2}\right)$ becomes infinite for $v=y$, solution (36) can be obtained by the following procedure:

(1) multiply both sides of the Equation (a) by $\mathrm{d} v / \sqrt{ }\left(u^{2}-v^{2}\right)$;

(2) integrate with respect to $v$ from 0 to $u$ after changing the order of integration in the double integral;

(3) take the derivative with respect to $u$ of both sides of the resulting equation;

(4) write $y$ instead of $u$. 
have a relation analogous to $(3)$ :

$$
f(y)=\frac{2}{\pi v_{b}} \frac{\sqrt{\left(v_{b}^{2}-y^{2}\right)}}{y} \frac{\partial}{\partial y} y \int_{0}^{y} \frac{v \varphi(v) \mathrm{d} v}{\sqrt{\left(y^{2}-v^{2}\right)}} .
$$

If the observed histogram is of high precision, the distribution $\varphi(v)$ can be obtained by (35), through numerical integration, provided that the empirical distribution has been approximated by a continuous function $f(y)$. On the other hand, owing to the uncertainty in the measures of the apparent rotational velocities or to the small number of the stars, it would be more correct to use (36) after assuming some form for $\varphi(v)$, with two parameters, for example of the type (5). In this case, for the numerical integration, it is more convenient to write (36) as:

$$
\begin{aligned}
f(y)=\frac{2 \sqrt{\left(v_{b}^{2}-y^{2}\right)}}{v_{b}}\left[\frac{y_{0}^{2}}{\sqrt{\left(y^{2}-y_{0}^{2}\right)}} \varphi\left(y_{0}\right)\right. & \\
& \left.+\int_{\sin ^{-1}\left(y_{0} / y\right)}^{\pi / 2} \sin \beta \frac{\partial}{\partial y}\left[y^{2} \varphi(y \sin \beta)\right] \mathrm{d} \beta\right],
\end{aligned}
$$

where $\sin \beta=v / y$, and the function $\varphi(v)$ must be defined different from zero only in $\left(y_{0}, v_{b}\right)$; further, $y_{0}$ ranges from 0 to $v_{b}$, depending on the interval $\left(0, y_{0}\right)$ where no apparent velocities have been observed.

It should be pointed out that the procedure contained in Equations (36) or (37) is analogous to that sketched in Section 1, but it has a different meaning: if we assume a function $\varphi(v)=0$ in $\left(0, y_{0}\right)$ and $\varphi(v) \neq 0$ in $\left(y_{0}, v_{b}\right)$, we can find a function $f(y) \neq 0$ in $\left(0, y_{0}\right)$ by means of (3), but this is impossible through (36) or (37); this fact does not imply the impossibility of observing rotational velocities smaller than $y_{0}$ for stars whose true rotational velocity is greater than $y_{0}$; it simply emphasizes that the present theory has been developed starting from the observations and gives a distribution $\varphi(v)$ different from zero in every interval where $f(y)$ does not vanish. It follows that any assumed $\varphi(v)$ has not necessarily a real physical meaning, unless we are able to find it, when the computed $f(y)$ has been fitted to the observed histogram; relations (36) and (37) are simply a mathematical tool in order to assign to the block-curve of the apparent ones, a continuous distribution of the true velocities which might be free from the accidental element contained in an empirical distribution, as far as possible.

\section{B. SPECIAL FORMS OF DISTRIBUTIONS}

With the purpose of comparing the results of the present statistical treatment with the ones derived under $\sin \alpha$, we consider in the following some special functions $f(y)$, which may conceivably represent particular observations;

(a) Suppose that the observed rotational velocities are distributed according to (8): it has already been shown (Section 1) that all stars rotate at the equatorial break-up if $\psi(\alpha)=\sin \alpha$. 
Presently, the frequency function $\psi(\alpha)$ is, by (8) and (19):

$$
\psi(\alpha)=-\sin \alpha \ln (\cos \alpha)
$$

which indicates that most of the stars are viewed nearly equatorially (Figure 5, curve a); hence the observed distribution will differ little from the true one, which turns out

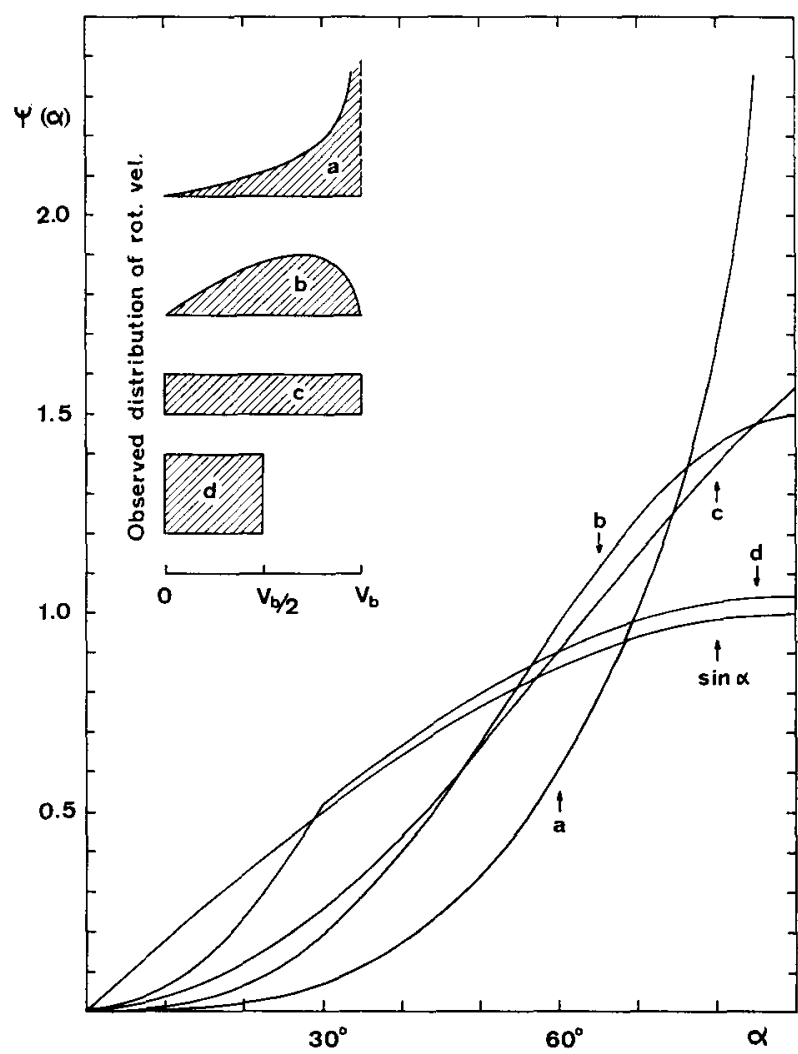

Fig. 5. The actual distribution function of the inclination $\alpha$ is shown for different observed distributions $(a, b, c, d)$ of the rotational velocities. The previous function $\sin \alpha$ is plotted for comparison.

to be:

$$
\varphi(v)=\frac{v_{b}^{2}}{v^{2} \sqrt{ }\left(v_{b}^{2}-v^{2}\right)} \sin ^{-1} \frac{v}{v_{b}}-\frac{1}{v} .
$$

The function (39) has been derived tediously by (35) and plotted in Figure 6a as a solid line. The difference between (39) and the $\delta$-solution (plotted as a dashed line in the figure) is remarkable. The result can be applied to the apparent rotational behaviour of the Be stars considered in Section 3; the observed cut-off is near $350 \mathrm{~km} / \mathrm{sec}$, if one star with $v \sin \alpha=400: \mathrm{km} / \mathrm{sec}$ is excluded. Since formula (8) with $v_{b}=350$ approximates satisfactorily the histogram ((a) and (c) of Figure 4), it has been believed 


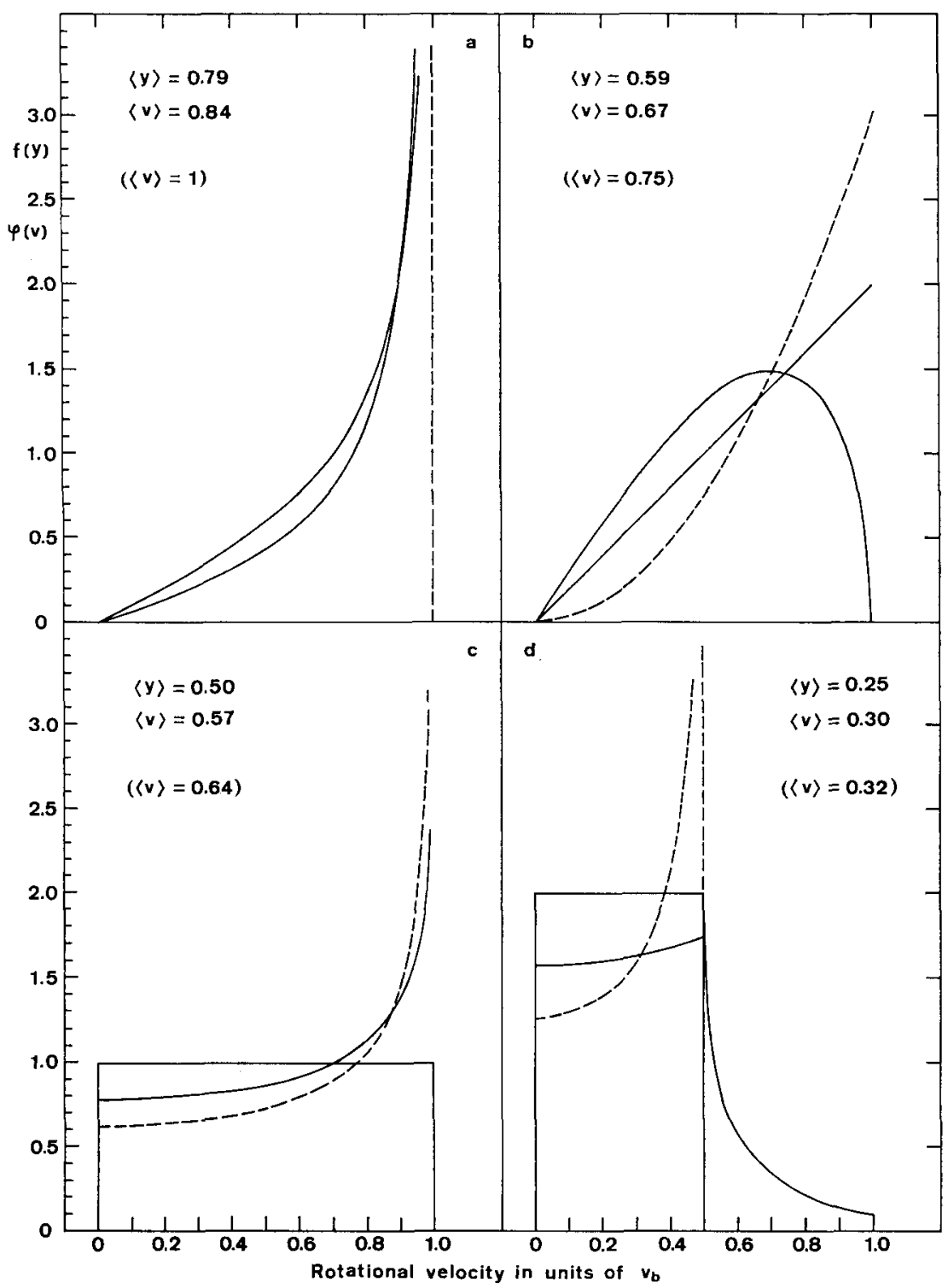

Fig. 6. The present distribution of the true rotational velocities (solid lines) is compared with that derived under $\sin \alpha$ (broken lines). The corresponding observed distributions $f(y)$, given by the appropriate solid tracings, are the same as in Figure $5(a, b, c, d)$. The mean of the apparent and true rotational velocities $(\langle y\rangle$ and $\langle v\rangle)$ are also indicated in units of the break-up limit $v_{b}$. In parentheses, the value of $\langle v\rangle$ has been computed by $\langle v\rangle=(4 / \pi)\langle y\rangle$. For a correct understanding of case d, see discussion in the text. 
that we are concerned with stars rotating probably at the same velocity of $350 \mathrm{~km} / \mathrm{sec}$ (Slettebak, 1966a; Huang, 1969).

If an effective upper limit to the true velocities near $350 \mathrm{~km} / \mathrm{sec}$ is assumed, because no velocity has been observed beyond it, we have actually no longer $\varphi(v)=\delta(v-350)$, but (39) where $v_{b}=350$; the mean true velocity is now $0.84 \times 350 \approx 300$ instead of $\approx 350 \mathrm{~km} / \mathrm{sec}$ and the spread around the mean becomes not negligible.

(b) Let the observed distribution be

$$
f(y)=\frac{3}{v_{b}^{3}} y \sqrt{ }\left(v_{b}^{2}-y^{2}\right) .
$$

The angle $\alpha$ turns out to be distributed according to:

$$
\psi(\alpha)=\frac{3}{2} \sin ^{3} \alpha
$$

(see Figure 5, curve $b$ ) and for the true velocities we have:

$$
\varphi(v)=2 v / v_{b}^{2} \quad 0 \leqslant v \leqslant v_{b}
$$

instead of:

$$
\varphi(v)=3 v^{2} / v_{b}^{3} \quad 0 \leqslant v \leqslant v_{b}
$$

which is the solution of the integral Equation (3).

The trend of both (42) and (43) is shown in Figure $6 \mathrm{~b}$ respectively by the solid straight line and the broken one: the solid curve represents the observations according to $(40)$.

Let us consider now an apparent distribution of the type (25); the function $\psi(\alpha)$ is given by (27) where it is now $N_{r}=N$ and $I_{r}=1 /\left(y_{r}-y_{r-1}\right)$; hereafter write $\Delta y_{r}$ instead of $y_{r}-y_{r-1}$.

Suppose that the apparent velocities have been accurately measured and the number of stars, $N$, is sufficiently large to believe that (25) may be a fairly good representation of the apparent rotational behaviour of our stars. If so, we attempt to use (35) to get $\varphi_{r}(v)$. We have:

$$
\begin{array}{ll}
\varphi_{r}(v)=\frac{v_{b}}{\Delta y_{r}} \int_{y_{r-1}}^{v} \frac{y^{2} \mathrm{~d} y}{v^{2} \sqrt{\left(v^{2}-y^{2}\right) \sqrt{ }\left(v_{b}^{2}-y^{2}\right)}} & y_{r-1} \leqslant v \leqslant y_{r} \\
\varphi_{r}(v)=\frac{v_{b}}{\Delta y_{r}} \int_{y_{r-1}}^{y_{r}} \frac{y^{2} \mathrm{~d} y}{v^{2} \sqrt{ }\left(v^{2}-y^{2}\right) \sqrt{\left(v_{b}^{2}-y^{2}\right)}} & y_{r} \leqslant v \leqslant v_{b}
\end{array}
$$

and, of course, $\varphi_{r}(v)=0$ for $0 \leqslant v \leqslant y_{r-1}$. The above integrals can be evaluated by means of elliptic integrals, as follows.

By (1) and the positions $v=x v_{b}$ and $y_{r}=s_{r} v_{b}$, (45) becomes:

$$
\Delta y_{r} \varphi_{r}\left(x v_{b}\right)=\int_{\sin ^{-1}}^{\sin _{\left(s_{r-1}-1\right.}^{-1}\left(s_{r} / x\right)} \frac{\sin ^{2} \alpha \mathrm{d} \alpha}{\sqrt{\left(1-x^{2} \sin ^{2} \alpha\right)}} \quad s_{r} \leqslant x \leqslant 1 .
$$


If the upper limit of the integration in (46) is set up equal to $\pi / 2$ we obtain (44).

Consider the Legendre integral $L_{r}(x)$, defined by:

$$
L_{r}(x)=\int_{0}^{\sin ^{-1}\left(s_{r} / x\right)} \frac{\sin ^{2} \alpha \mathrm{d} \alpha}{\left.\sqrt{\left(1-x^{2}\right.}-\sin ^{2} \alpha\right)}
$$

and the following elliptic integrals of first and second kind, respectively:

$$
\begin{aligned}
& F_{r}(x)=\int_{0}^{\sin ^{-1}\left(s_{r} / x\right)}\left(1-x^{2} \sin ^{2} \alpha\right)^{-1 / 2} \mathrm{~d} \alpha \\
& E_{r}(x)=\int_{0}^{\sin ^{-1}\left(s_{r} / x\right)}\left(1-x^{2} \sin ^{2} \alpha\right)^{1 / 2} \mathrm{~d} \alpha .
\end{aligned}
$$

Integrals (48) and (49) can be readily found in mathematical tables. In terms of (48) and (49), the Legendre integral (47) can be written:

$$
L_{r}(x)=x^{-2}\left[F_{r}(x)-E_{r}(x)\right] \text {. }
$$

If the upper limit of integration in (47), (48), (49) is set up equal to $\pi / 2$, formula (50) yields the complete Legendre integral $L_{c}(x)$, so that (44) and (45) can be evaluated by means of:

$$
\begin{array}{lr}
\Delta y_{r} \varphi_{r}\left(x v_{b}\right)=L_{c}(x)-L_{r-1}(x) & s_{r-1} \leqslant x \leqslant s_{r} \\
\Delta y_{r} \varphi_{r}\left(x v_{b}\right)=L_{r}(x)-L_{r-1}(x) & s_{r} \leqslant x \leqslant 1 .
\end{array}
$$

Let us consider now two particular cases of distributions (25):

(c) $y_{r}=v_{b}, \quad y_{r-1}=0$

(d) $\quad y_{r}=y_{0} \neq v_{b}, \quad y_{r-1}=0$.

In case (c) we soon find that the rotational axes are distributed according to $\alpha \sin \alpha$ (Figure 5, curve c) and, from the preceding analysis, the function which governs the true velocities turns out to be:

$$
v_{b} \varphi(v)=L_{c}\left(v / v_{b}\right)
$$

If (2) is assumed to represent the distribution of $\alpha, \varphi(v)$ can be obtained by (4) using in it $f(y)=1 / v_{b}$, resulting in:

$$
\varphi(v)=\frac{2}{\pi} \frac{1}{\sqrt{\left(v_{b}^{2}-v^{2}\right)}} .
$$

The difference between (53) and (54) is small (Figure 6c); for instance, according to (54) $32 \%$ of the stars have a rotational velocity between 0 and $0.5 v_{b}$, while by (53) we have $40 \%$ in the same interval.

The special distribution (d) requires particular attention if we treat it by (51) and (52). 
The analytical form of $\psi(\alpha)$ is:

$$
\begin{aligned}
\psi(\alpha) & =\frac{v_{b}}{y_{0}} \alpha \sin \alpha & & 0 \leqslant \alpha \leqslant \theta_{0} \\
& =\frac{v_{b}}{y_{0}} \theta_{0} \sin \alpha & & \theta_{0} \leqslant \alpha \leqslant \pi / 2
\end{aligned}
$$

and the distribution of the true velocities, through (51) and (52), where it is now $\Delta y_{r}=y_{0}$, is given by:

$$
\begin{array}{rlrl}
\varphi(v) & =y_{0}^{-1} L_{c}\left(v / v_{b}\right) & 0 & \leqslant v \leqslant y_{0} \\
& =y_{0}^{-1} L\left(\frac{v}{v_{b}} ; \sin ^{-1} \frac{y_{0}}{v}\right) & y_{0} \leqslant v \leqslant v_{b} .
\end{array}
$$

Let us fix the value of $y_{0}$ to be $v_{b} / 2$; then (55) differs little from $\sin \alpha$ (Figure 5 , curve d) so that we expect that $\varphi(v)$ derived by (3) will differ little from (56); the situation is apparently quite different since it can be easily verified that the following function:

$$
\begin{aligned}
\varphi(v) & =\frac{2}{\pi}-\frac{1}{\sqrt{\left(y_{0}^{2}-v^{2}\right)}} & & 0 \leqslant v \leqslant y_{0} \\
& =0 & & y_{0}<v \leqslant v_{b}
\end{aligned}
$$

is the solution of the integral Equation (3) for the particular $f(y)$ under discussion (distributions (56) and (57) have been plotted in Figure 6d assuming $y_{0}=v_{b} / 2$ ).

Relation (57) excludes the existence of stars rotating beyond $y_{0}$ while one would expect to find them; on the other hand by (56) there would be $\approx 25 \%$ of the stars with a velocity in $(0.5,1)$ but none of them has been observed near the equator; moreover, only stars with $v \leqslant 0.5$ have been observed equator-on. Such a result is clearly unreliable, if one does not pay attention to the meaning of the observed distribution. Consider the following:

If $f(y)=1 / y_{0}$ is the result of observations in a very large sample of stars, it is very likely that the size of the sample is sufficient to make $y_{0}$ the effective upper-limit to the true velocities; hence case $\mathrm{d}$ degenerates into case $\mathrm{c}$ and any strangeness drops.

Otherwise, when the number of stars observed is not large enough, the histogram $f^{\prime}(y)=1 / y_{0}$ must be considered necessarily as a rough empirical distribution. Then it would be more correct to fit it to a continuous function by means of (36) or (37), and the distribution (56) may indicate fairly well the form of the function $\varphi(v)$, that one must assume; an analogous procedure gives in this case a reliable true distribution under (2) (as was explained in Section 1), that would result completely different from (57) and similar to (56).

It does not seem, however, that (56) gives information about the distribution of the true rotational velocities of less accuracy than the observed histogram does about the apparent ones; using (56) we have the advantage of getting almost immediately an idea of the rotational behaviour of our stars: for instance, in the case shown in Figure $6 \mathrm{~d}$, we are not far from the probabilistic truth if $\approx 25 \%$ of the stars are predicted 


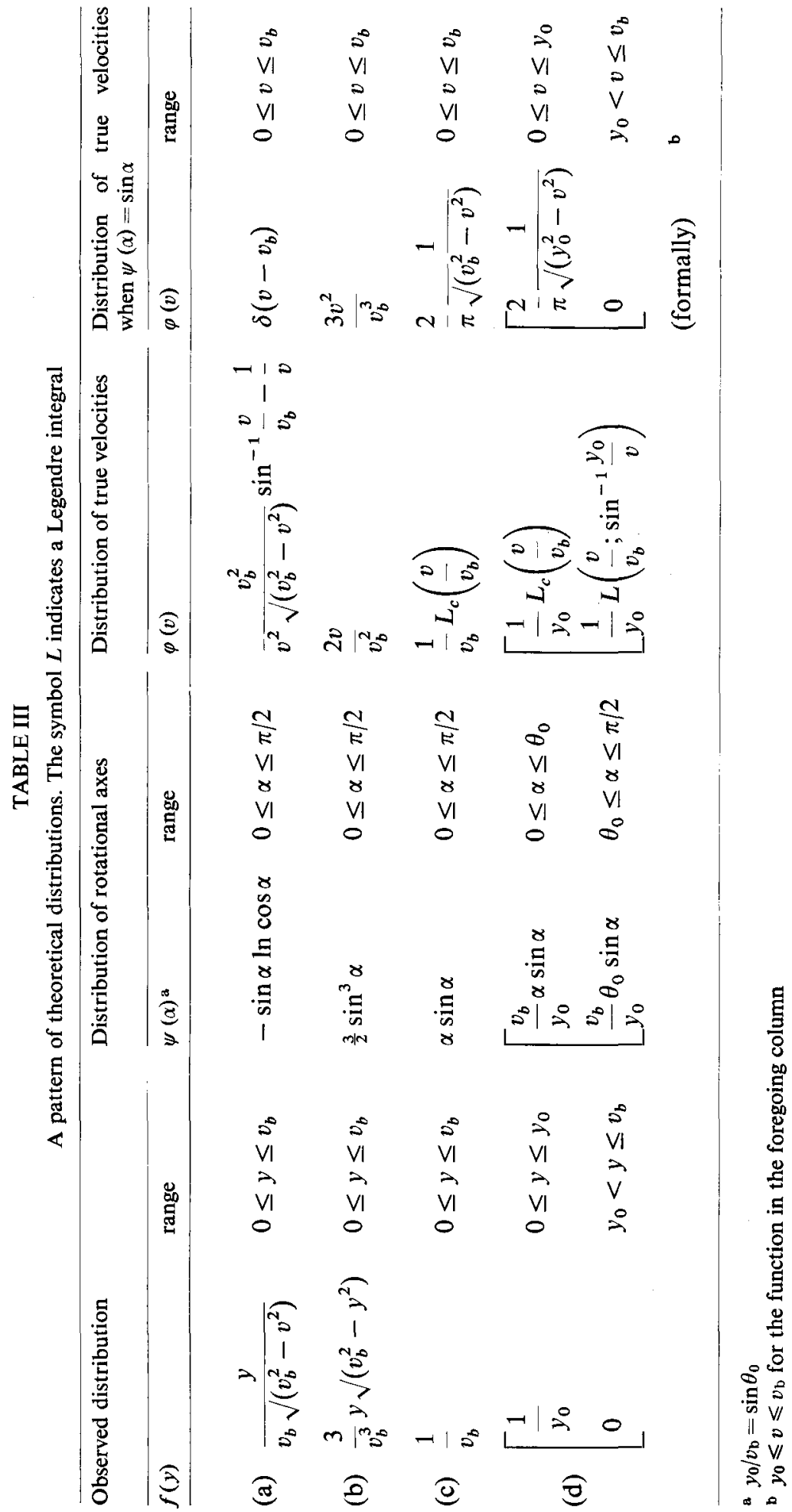


to rotate in $(0.5,1)$ and $\approx 75 \%$ in $(0,0.5)$; it is not difficult to infer that an elegant distribution $\varphi(v)$, derived by the formally correct procedure will yield practically the same expectation.

All the frequency functions discussed so far, have been collected in Table III.

\section{A Method of Getting a Rough Distribution $\varphi(v)$ from the Histogram of the Apparent Rotational Velocities}

The correct procedure to derive a true distribution $\varphi(v)$ has been discussed in Section 4. However, (36) or (37) requires the tabulation of a set of functions $\varphi(v)$, each truncated at $v_{b}$, in order to fit any observed histogram. Alternatively any observed histogram must be approximated by a suitable continuous function $f(y)$ in order to integrate (35).

This procedure may be tedious and time consuming; in the following we suggest a means to get an approximate trend of $\varphi(v)$ by simple numerical calculations, starting from the case (d) considered in the foregoing section, which makes it possible to treat each block of a histogram by formulae (51) and (52).

Let us take a histogram of the type (26) and call $\varphi_{r}\left(x v_{b}\right)$ the distribution of the true velocities $v=x v_{b}$ for the stars which have been observed between $y_{r-1}$ and $y_{r}$.

Analogously to what has been done in Section 3, the total distribution $\varphi\left(x v_{b}\right)$ is:

$$
\varphi\left(x v_{b}\right)=\sum_{r} I_{r} \Delta y_{r} \varphi_{r}\left(x v_{b}\right) .
$$

When (51) and (52) have been used in (58), we can write for every $x$ ranging between $s_{r-1}$ and $s_{r}$ :

$$
\varphi\left(x v_{b}\right)_{r-1, r}=I_{r} L_{c}(x)-\sum_{1}^{r-1}{ }_{k}\left(I_{k+1}-I_{k}\right) L_{k}(x) .
$$

Although the trend of (59) may represent the distribution of the rotational velocities, it is not of very practical use: for our purpose it is sufficient to get the mean value of $\varphi(v)$ in every interval $\left(y_{r-1}, y_{r}\right)$, that is:

$$
\langle\varphi\rangle_{r-1, r}=\left(s_{r}-s_{r-1}\right)^{-1} \int_{s_{r-1}}^{s_{r}} \varphi\left(x v_{b}\right)_{r-1, r} \mathrm{~d} x .
$$

After the calculation has been performed in (60) by simple integration of the Legendre integrals with respect to $x$, we find:

$$
\begin{aligned}
\langle\varphi\rangle_{r-1, r}=\frac{I_{r}}{s_{r}-S_{r-1}} & \left(T_{r r}-T_{r-1 r-1}\right)+ \\
& \quad-\left(s_{r}-s_{r-1}\right)^{-1} \sum_{1}^{r-1} k_{k}\left(I_{k+1}-I_{k}\right)\left(T_{k r}-T_{k r-1}\right),
\end{aligned}
$$

where:

$$
T_{k r}=s_{r}^{-1} E\left(\beta_{r}^{k} ; \theta_{r}\right)-s_{r}^{-1}\left(1-s_{r}^{2}\right) F\left(\beta_{r}^{k} ; \theta_{r}\right)
$$


The elliptic integral $F$ and $E$, already defined in Section 4, are given now by:

$$
\begin{aligned}
& F\left(\beta_{r}^{k} ; \theta_{r}\right)=\int_{0}^{\beta_{r^{k}}}\left(1-\sin ^{2} \theta_{r} \sin ^{2} \alpha\right)^{-1 / 2} \mathrm{~d} \alpha \\
& E\left(\beta_{r}^{k} ; \theta_{r}\right)=\int_{0}^{\beta_{r^{k}}}\left(1-\sin ^{2} \theta_{r} \sin ^{2} \alpha\right)^{1 / 2} \mathrm{~d} \alpha,
\end{aligned}
$$

where:

$$
\begin{aligned}
& \theta_{r}=\sin ^{-1} s_{r}=\sin ^{-1}\left(y_{r} / v_{b}\right) \\
& \beta_{r}^{k}=\sin ^{-1}\left(s_{k} / s_{r}\right)=\sin ^{-1}\left(y_{k} / y_{r}\right) .
\end{aligned}
$$

In (63) and (64) we have written $\sin ^{2} \theta_{r}$ instead of $s_{r}^{2}$ since in mathematical tables elliptic integrals may be tabulated for a set of values of the modular angle $\theta_{r}$.

As an example of the application of the foregoing formulae (61)-(66), let us consider the Be stars partially analysed in Section 3; we may start from the histogram (b) (Table I and Figure 3), assuming again a break-up velocity of $450 \mathrm{~km} / \mathrm{sec}$ for all stars, and select the first interval between 0 and $100 \mathrm{~km} / \mathrm{sec}$ and the following ones of $50 \mathrm{~km} / \mathrm{sec}$ in size up to $450 \mathrm{~km} / \mathrm{sec}$.

If one looks, for instance, for the mean of $\varphi(v)$ between 100 and $150 \mathrm{~km} / \mathrm{sec}$, (61) gives:

$$
\langle\varphi\rangle_{12}=\frac{I_{2}}{s_{2}-s_{1}}\left(T_{22}-T_{11}\right)-\frac{1}{s_{2}-s_{1}}\left(I_{2}-I_{1}\right)\left(T_{12}-T_{11}\right),
$$

where, according to (62)-(66), it is :

$$
\begin{aligned}
& T_{11}=s_{1}^{-1} E\left(\pi / 2 ; \theta_{1}\right)-s_{1}^{-1}\left(1-s_{1}^{2}\right) F\left(\pi / 2 ; \theta_{1}\right) \\
& T_{12}=s_{2}^{-1} E\left(\beta_{2}^{1} ; \theta_{2}\right)-s_{2}^{-1}\left(1-s_{2}^{2}\right) F\left(\beta_{2}^{1} ; \theta_{2}\right) \\
& T_{22}=s_{2}^{-1} E\left(\pi / 2 ; \theta_{2}\right)-s_{2}^{-1}\left(1-s_{2}^{2}\right) F\left(\pi / 2 ; \theta_{2}\right) .
\end{aligned}
$$

The numerical pattern is as follows:

$$
\begin{aligned}
& I_{1}=0.048 \times 10^{-2} \\
& I_{2}=0.119 \times 10^{-2} \\
& s_{1}=100 / 450 \\
& s_{2}=150 / 450 \\
& \theta_{1}=\sin ^{-1} s_{1} \approx 13^{\circ} \\
& \theta_{2}=\sin ^{-1} s_{2} \approx 19^{\circ} .5 \\
& \beta_{2}^{1}=\sin ^{-1}\left(s_{1} / s_{2}\right) \approx 42^{\circ} .
\end{aligned}
$$

By interpolation in mathematical tables we find $F\left(90^{\circ} ; 13^{\circ}\right), F\left(90^{\circ} ; 19^{\circ} .5\right), F\left(42^{\circ}\right.$; $\left.19^{\circ} .5\right)$ and the corresponding elliptic integrals of second kind $E$; finally we get:

$$
\langle\varphi\rangle_{12} \approx 0.075 \times 10^{-2} \text {. }
$$




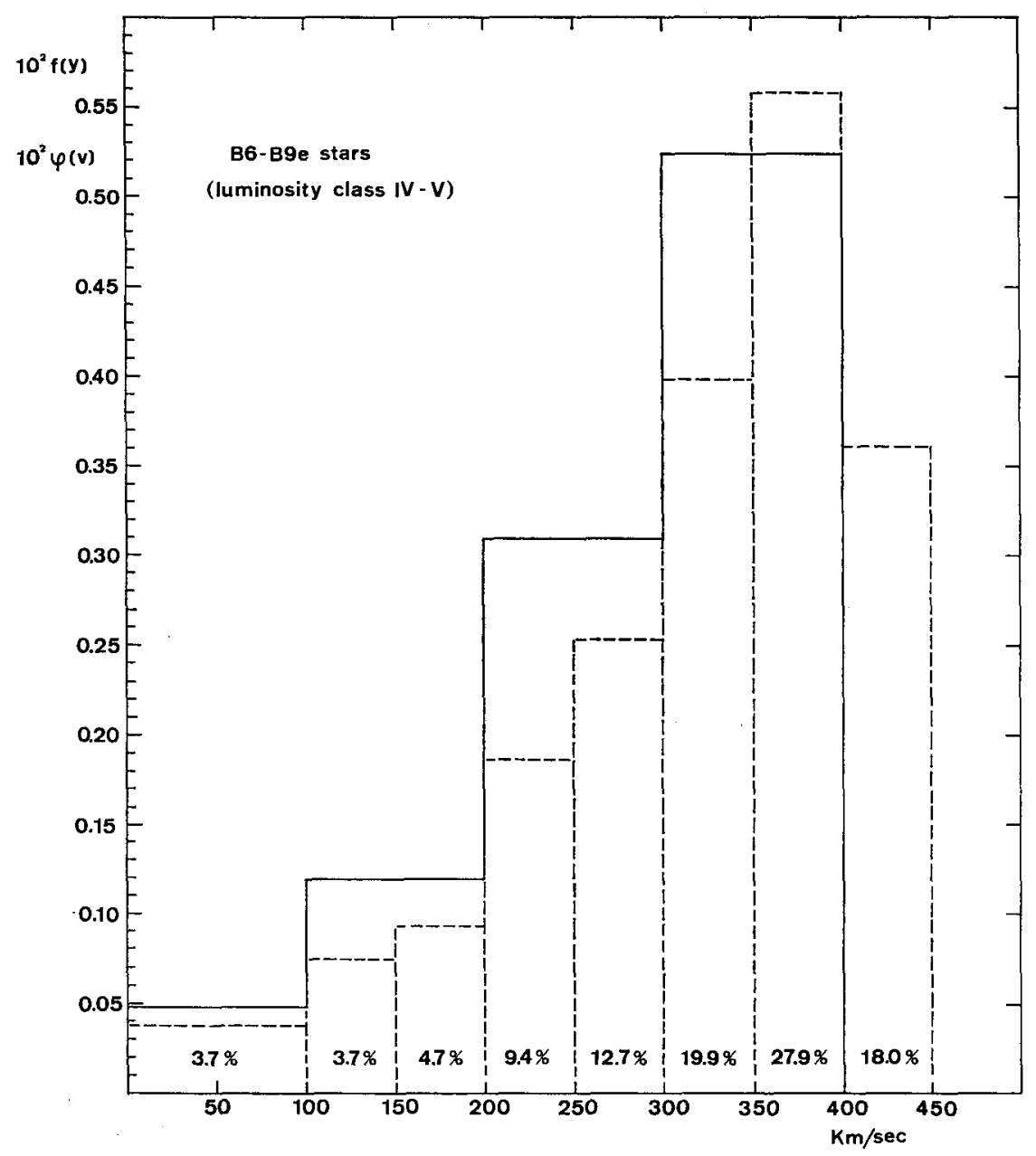

Fig. 7. Approximate trend of the distribution of the rotational velocities $v$ for Be stars, given in the form of a histogram (broken tracing). The solid tracing is the histogram of the apparent velocities $y=v \sin \alpha$ (case $b$ of Figure 4). The percentage of stars in each interval of the 'true histogram' is shown. The cut-off is assumed to be at $450 \mathrm{~km} / \mathrm{sec}$.

The whole histogram of the true velocities is shown in Figure 7 by the broken tracing; the percentage of stars in each interval has also been indicated. It appears that a Be star does not need to be a rapid rotator; if the usual assumption of equatorial envelopes yielding a shell spectrum is correct, then shell stars are viewed nearly equator-on; if so, the statistical method so far suggested would explain the observation of Be stars with a shell spectrum at velocities lower than $300 \mathrm{~km} / \mathrm{sec}$ (Slettebak, 1966a), a fact that a $\delta$-solution could not explain.

It is useful to notice that the existence of intrinsically slow rotators among the Be stars has already been suggested on an observational basis by some authors (Schild, 1966; Deeming and Walker, 1967). 


\section{Concluding Remarks}

In Sections 2-4 the statistical method of investigating the rotational behaviour of a group of stars has been established on the basis of the observations and the existence of an upper limit to the true velocities.

It may be useful to emphasize the kind of problem we are dealing with. Suppose we have a sample of bodies of known rotational velocity and spread them randomly in space. If one asks for the expectation of the apparent rotational velocity $f(y)$, the solution is just (3) where $\varphi(v)$ is known. The actual problem is quite different: apparent velocities are observed and a guess as to the distribution of the true velocities is required; the best estimate is obtained by the method developed in this paper. The usual procedure of considering valid a law when its consequences, derived under admissible general hypothesis, have been observed, cannot be applied to rotational velocities.

The present method depends on the selection of the upper limit and one must operate carefully; consider, for instance, the following: it is well known that Ap stars are observed to be slow rotators; suppose that we are dealing with a sample for which the maximum apparent velocity is $100 \mathrm{~km} / \mathrm{sec}$ : if an upper limit of, say, $400 \mathrm{~km} / \mathrm{sec}$ is assumed for A-stars (Figure 3), there is no difference in practice between using the earlier treatment (with $\sin \alpha$ ) or the present one; if, however, Ap stars are considered to be intrinsically slow rotators, it may be quite correct to take the observed maximum as the effective upper limit so that the use of the present method becomes important.

Finally, let us consider briefly again the approximate method of Section 5: since $T_{k r}$ depends only on the selection of the intervals $\left(s_{r-1}, s_{r}\right)$, one can construct once for all a square matrix:

$$
T=\left(\begin{array}{llllll}
T_{11} & T_{12} & T_{13} & \cdot & \cdot & T_{1 n} \\
0 & T_{22} & T_{23} & \cdot & . & T_{2 n} \\
0 & 0 & T_{33} & \cdot & \cdot & T_{3 n} \\
\cdot & \cdot & \cdot & \cdot & \cdot & \cdot \\
\cdot & \cdot & \cdot & \cdot & \cdot & T_{n n}
\end{array}\right)
$$

where $n$ is the number of intervals. Taking $n=20$, for instance, we have the possibility by $T$ and (61) of transforming immediately a large variety of observed histograms yielding results of sufficient accuracy, within the errors by which apparent velocities have been determined.

\section{Acknowledgements}

I am grateful to Drs. R. Barbon, M. Capaccioli and T. Minelli for helpful discussions. I wish to thank also Mr. M. Franceschi, who did most of the numerical computation and drawings.

\section{References}

Chandrasekhar, S. and Münch, G.: 1950, Astrophys. J. 111, 142.

Deeming, T. J. and Walker, G. A. H.: 1967, Nature, February 4, 479. 
Huang, S. S.: 1953, Astrophys. J. 118, 285.

Huang, S. S.: 1969, Vistas in Astronomy, vol. 11 (ed. by A. Beer), Pergamon Press, p. 217.

Schild, R. E.: 1966, Astrophys. J. 146, 142.

Shajn, G. and Struve, O.: 1929, Monthly Notices Roy. Astron. Soc. 89, 222.

Slettebak, A.: 1949, Astrophys. J. 110, 498.

Slettebak, A.: 1966a, Astrophys. J. 145, 121.

Slettebak, A.: 1966b, Astrophys. J. 145, 126.

Trumpler, R. J. and Weaver, H. F.: 1953, Statistical Astronomy, University of California Press,

Berkeley and Los Angeles.

\section{Discussion}

Steinitz: Would an observer in some other location in space obtain the same $\psi(\alpha)$ ?

Bernacca: In general, he will obtain a different $\psi(\alpha)$. Assume for a moment that the axes of Be stars are nearly perpendicular to the galactic plane. For a sample of such stars, all rotating near the break-up velocity, an observer located on the galactic plane and far from the sample would derive $\psi(\alpha) \simeq \delta(\alpha-\pi / 2)$. On the other hand, an observer located far from the galaxy in the direction of the galactic pole would always measure small $v \sin \alpha$, say $\leqslant 100 \mathrm{~km} / \mathrm{sec}$. If he thinks that Be stars are normally very rapid rotators and recognizes pole-on characteristics in all stars, he might conclude that $\psi(\alpha) \simeq \delta(\alpha)$. Otherwise he may assume that the Be phenomenon is independent of rotation and take $100 \mathrm{~km} / \mathrm{sec}$ as the effective upper limit because no larger velocities have been observed: $\psi(\alpha)$ would turn out to be different again, depending on $f(y)$.

Steinitz: Would you agree that some of our classification schemes are aspect dependent?

Bernacca: I agree.

Collins: How sensitive are your results to the choice of $V_{b}$ (i.e., the critical velocity)?

Bernacca: I can give an answer, at the moment, only for a sample of stars with the same critical velocity. The choice of $V_{b}$ affects mostly the mean square deviations of a true distribution; however, if you have a large sample of stars, the upper limit $V_{b}$ cannot be too far from the maximum observed apparent velocity, unless you are aware of selection effects. If the number of stars is small, any statistical method will yield unreliable results, of course. Let me point out that $V_{b}$ is not necessarily the critical velocity, but any other upper limit which can be recognized or assumed reasonably. 